\title{
BMJ Global Health Implementing a mobile diagnostic unit to increase access to imaging and laboratory services in western Kenya
}

\author{
Roshni Dhoot, ${ }^{1}$ John M Humphrey, ${ }^{2,3}$ Patrick O'Meara, ${ }^{3,4}$ Adrian Gardner, ${ }^{2,3}$ \\ Clement J McDonald, ${ }^{5}$ Kelvin Ogot, ${ }^{6}$ Sameer Antani, ${ }^{5}$ Joseph Abuya, ${ }^{7}$ Marc Kohli ${ }^{8}$
}

To cite: Dhoot R, Humphrey JM, O'Meara P, et al. Implementing a mobile diagnostic unit to increase access to imaging and laboratory services in western Kenya. BMJ Glob Health 2018;3:e000947. doi:10.1136/ bmjgh-2018-000947

Handling editor Seye Abimbola

Received 11 May 2018

Revised 8 August 2018

Accepted 9 August 2018

Check for updates

(C) Author(s) (or their employer(s)) 2018. Re-use permitted under CC BY-NC. No commercial re-use. See rights and permissions. Published by BMJ.

${ }^{1}$ School of Medicine, Indiana University, Indianapolis, Indiana USA

${ }^{2}$ Department of Medicine, Indiana University, School of Medicine, Indianapolis, Indiana, USA

${ }^{3}$ Academic Model Providing Access to Healthcare (AMPATH), Eldoret, Kenya

${ }^{4}$ Wataalamu Repair and Maintenance, Eldoret, Kenya ${ }^{5}$ National Library of Medicine, National Institutes of Health, Bethesda, Maryland, USA ${ }^{6}$ Radiology and Imaging Department, Moi Teaching and Referral Hospital, Eldoret, Kenya ${ }^{7}$ Department of Radiology, Moi University, Moi University, College of Health Sciences, School of Medicine, Eldoret, Kenya

${ }^{8}$ Radiology and Biomedical Imaging, University of California San Francisco, San Francisco, California, USA

Correspondence to

Dr Marc Kohli;

marc.kohli@ucsf.edu

\section{ABSTRACT}

Access to basic imaging and laboratory services remains a major challenge in rural, resource-limited settings in subSaharan Africa. In 2016, the Academic Model Providing Access to Healthcare programme in western Kenya implemented a mobile diagnostic unit (MDU) outfitted with a generator-powered $\mathrm{X}$-ray machine and basic laboratory tests to address the lack of these services at rural, lowresource, public health facilities. The objective of this paper is to describe the design, implementation, preliminary impact and operational challenges of the MDU in western Kenya. Since implementing the MDU at seven rural health facilities serving a catchment of over half a million people, over 4500 chest radiographs have been performed, with one or more abnormalities detected in approximately $30 \%$ of radiographs. We observed favorable feedback and uptake of MDU services by healthcare workers and patients. However, various operational challenges in the design and construction of the MDU and the transmission and reporting of radiographs in remote areas were encountered. Our experience supports the feasibility of deploying an MDU to increase access to basic radiology and laboratory services in rural, resource-limited settings.

\section{BACKGROUND}

Access to basic imaging and laboratory services remains a major challenge in rural, resource-limited settings in sub-Saharan Africa. In Kenya, $74 \%$ of the population live in rural areas with low population density, serviced by health outposts that lack basic radiology equipment, trained radiologists and laboratory tests. ${ }^{1}$ Moreover, 90\% of Kenya's radiologists are estimated to be working in urban settings, with $76 \%$ (80 of 105) of radiologists in Kenya concentrated in three major cities. ${ }^{2}$ Similar disparities in access to radiographic imaging services exist elsewhere in East Africa. In Uganda, for example, $56 \%$ of patients requiring imaging receive such services in urban areas compared with $10 \%-13 \%$ of patients in rural areas, and an estimated $90 \%$ of radiographs performed do not have an accompanying imaging report. ${ }^{3}$

\section{Summary box}

Access to basic imaging and laboratory services in rural, resource-limited settings in sub-Saharan Africa is limited, particularly in areas facing a high burden of tuberculosis.

- In 2016, the Academic Model Providing Access to Healthcare programme implemented a mobile diagnostic unit (MDU) with a generator and wireless digital radiography which has taken over $4500 \mathrm{X}$-rays to date, detecting abnormalities in approximately $30 \%$ of images.

- Benefits of the MDU include high rates of detection of abnormal X-ray findings, improved patient access to radiology services and increased patient engagement; transmission of primary radiology reports is a challenge.

- Implementing a mobile vehicle equipped with X-ray capabilities and basic laboratory services is a feasible and potentially scalable means to increase access to these services in resource-limited areas.

The optimal strategy to increase access to radiographic imaging in these settings is not well understood.

Access to radiology services in resource-constrained settings in sub-Saharan Africa is particularly critical when one considers the unmeasured burden of tuberculosis (TB) and the major role of chest radiography in TB detection and control. ${ }^{4}$ Kenya is among 30 countries that together carry the world's highest country-level burdens of TB. ${ }^{5}$ The 2016 Kenya TB Prevalence Survey found a national TB prevalence of 558 cases per 100 000 people, more than twice that of previous estimates by WHO. ${ }^{5}$ Moreover, more than $40 \%$ of $\mathrm{TB}$ cases remained undetected and untreated. TB case detection has been shown to decrease with increasing distance from a hospital, with patients living further from the facility at higher risk of more severe TB disease. ${ }^{6}$ Chest radiography is recommended by $\mathrm{WHO}$ as a screening tool for $\mathrm{TB}$ given 
its high sensitivity. ${ }^{4}$ However, the lack of radiographic imaging capacity in rural areas remains a major barrier to implementation of this recommendation. Several studies have described the use of mobile radiography units to address the burden of TB in rural areas. ${ }^{6-8}$

The Academic Model Providing Access to Healthcare (AMPATH) programme is a consortium of North American Universities working in collaboration with the Kenya Ministry of Health (MOH), Moi University College of Health Sciences, and Moi Teaching and Referral Hospital in western Kenya. ${ }^{9}$ By engaging in the delivery of health-promoting services to individuals and populations in low-income environments, AMPATH and its partners aim to build health systems in the public sector that deliver and sustain essential healthcare services, develop human capacity through training and education, advance research, eliminate health disparities and mutually strengthen institutions. As part of this population health strategy, AMPATH designed and implemented a truck called the mobile diagnostic unit (MDU) that was outfitted with an X-ray generator, computed radiography system and gasoline generator. The MDU was first operationalised in September of 2014. In 2016, the MDU was augmented with digital radiography and laboratory testing. Following these upgrades, the MDU has travelled daily to rural health outposts within the AMPATH network to deliver radiology and laboratory services. The objective of this paper is to describe the design, implementation, preliminary impact, and operational challenges of the AMPATH MDU in western Kenya.

\section{DESIGN AND OPERATION AMPATH program}

AMPATH started an HIV care and treatment program in 2001 and has since expanded to about 500 clinical sites in western Kenya that have enrolled over 200000 patients with HIV with approximately 1500 new patients enrolling each month. ${ }^{10}$ AMPATH also supports MOH delivery of primary healthcare, chronic disease care, specialty care and supports community activities including community-run microfinance groups to help address socioeconomic determinants of health.

\section{Setting}

AMPATH is the President's Emergency Plan for AIDS Relief-supported HIV care implementing partner in four of the seven sub-counties of Busia County, namely Butula, Bunyala, Matayos and Teso North. According to the most recent census (2009), Busia County is home to an estimated 743946 people. ${ }^{11}$ The HIV prevalence in the county is $6.8 \%$ and the poverty level is $66 \% .^{12}$ Although the AMPATH-supported facilities in Busia County serve a catchment area of half a million people, they are poorly resourced, and many do not offer basic laboratory or radiology diagnostic services. Illustrating this, the major county hospital in Busia town, the capital of Busia County, had a digital X-ray unit installed in 2016 after a period of
14 months without any functional X-ray unit. Several of the facilities serviced by the MDU have only intermittent access to electricity and Internet.

Within the four sub-counties, AMPATH supports HIV service delivery in $66 \mathrm{MOH}$ facilities including one county referral, three sub-county hospitals, eight health centres, 53 dispensaries and five private owned/faith-based facilities. Two of these facilities, the county referral hospital and one sub-county hospital, currently have functional X-ray facilities. The MDU currently travels to seven AMPATH-supported MOH facilities in Busia County on the westernmost border of Kenya: Angurai, Bumala A, Bumala B, Khunyangu, Matayos, Mukhobola and Port Victoria (figure 1). On average, it takes approximately 2 hours to travel from the communities AMPATH serves to Busia County hospital, at a cost of between 500 and 1500 Kenyan shillings (US\$5-15) by public transport such as a motorbike, mini-bus or shared taxi.

\section{Design}

The MDU design, customisation and installation were performed locally. The MDU is a standard Isuzu canter chassis (3-4 tonnes) with a custom-designed, insulated/ refrigeration box body, complete with integrally welded, mild steel radiation shielding and washable interior plastic surfaces. The body was further customised with elements including a power control system, anchoring points for equipment, roll-out drawer-mounted AC generation and fuel can. The design incorporates several elements aimed at safety including a negative pressure ventilation system, multipoint redundant electrical grounding, strategically placed mild steel radiation shielding, ample interior space and lighting, patient privacy curtain and an integrated ramp for non-ambulatory patients (figure 2). Implementing a high-frequency X-ray generator in a mobile capacity, free from mains power, was challenging. Due to a lack of detailed information regarding peak power consumption of the X-ray generator, the $\mathrm{AC}$ power generator was initially undersized, and the X-ray generator would not consistently fire without power alarms and failed exposures. After extensive testing, this was mitigated by engineering and installing a low-cost power factor correction device.

\section{Cost}

Implementation and monthly maintenance costs for the mobile diagnostic unit can be found below in tables 1 and 2.

\section{Operation}

A radiographer employed by the $\mathrm{MOH}$ in Busia County performs the studies. The schedule for the MDU is determined monthly by a Busia County-based coordinator of AMPATH services and disseminated to the clinics at the beginning of the month. The MDU travels to each site at least twice per month with additional visits to sites according to the size of their patient population and need for services. Patients are scheduled for 


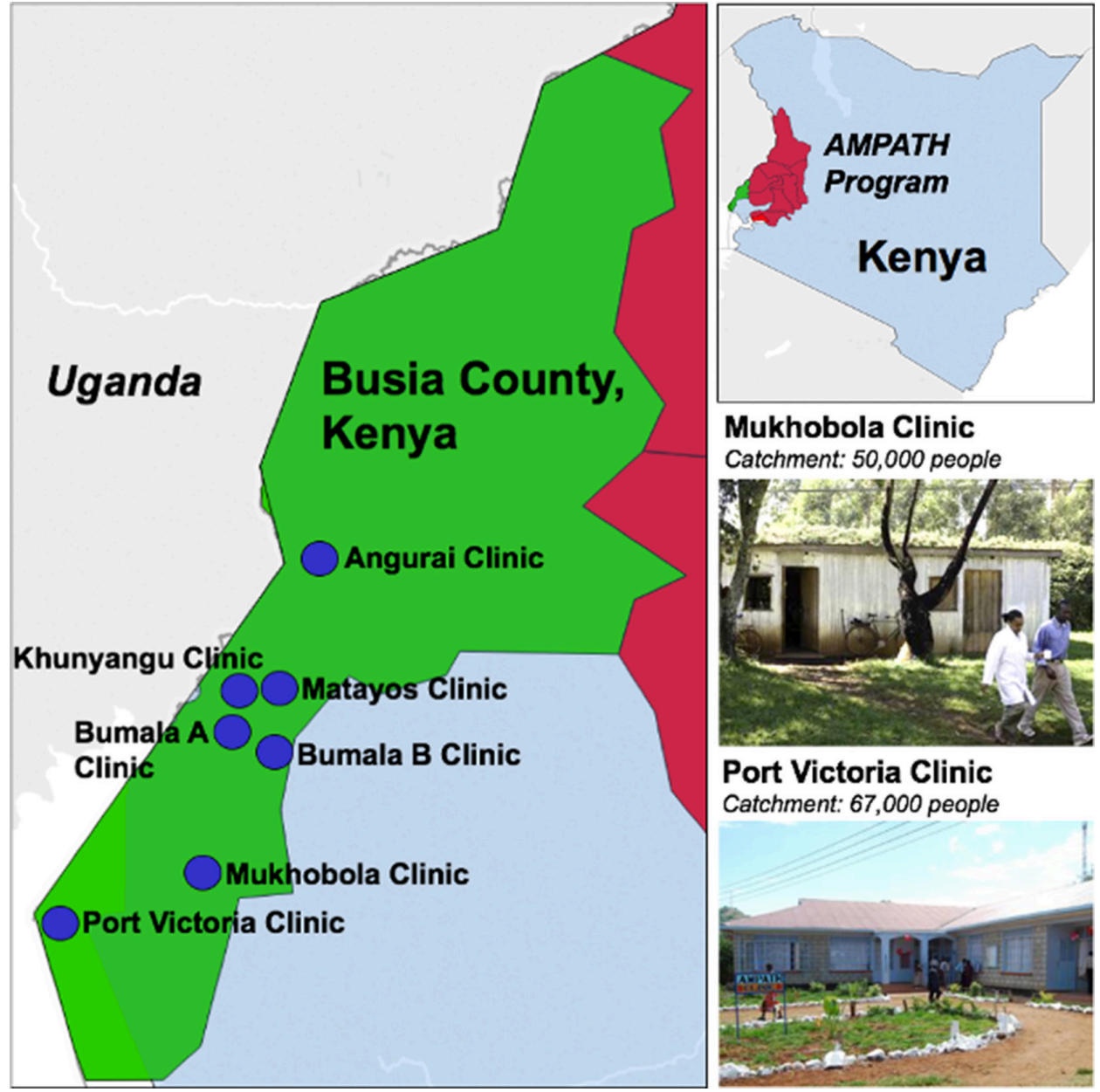

Figure 1 Locations of Academic Model Providing Access to Healthcare (AMPATH) clinics served by the mobile diagnostic unit (MDU) in Busia County, Kenya. The red shaded area of the map of Kenya indicates the counties served by the AMPATH programme in the western region of the country. The green shading of the map on the left shows Busia County, where the MDU operates; blue circles indicate the locations of clinics served by the MDU. Photos of two of these clinics' areas are shown in the lower right of the figure, along with the clinic names and catchment size.

a radiograph or laboratory test by the clinician at each facility and asked to return to clinic on the next MDU day. X-rays are performed for tuberculosis screening purposes in HIV-positive patients per WHO and Kenya MOH guidelines, and for diagnostic purposes in patients with respiratory symptoms and offered free of charge. ${ }^{1314}$ HIV-infected patients in Kenya who are asymptomatic are also routinely screened for serum cryptococcal antigen if their $\mathrm{CD} 4^{+}$count is $\leq 100$ cells $/ \mu \mathrm{L}$. If the radiographer notes the image to be grossly abnormal, he notifies the clinician immediately to review the image and determine any necessary management course. At the end of the day, radiographs are copied onto a $\mathrm{CD}$, which is maintained at each site for review and recordkeeping. Radiographs were originally acquired with a computed radiography system (Kodak POC 140), which was replaced with a wireless digital radiography (Carestream) system in 2016 to save operator time, reduce space requirements and decrease electrical load. Images were originally transported to a central archive via DVDs burned from the computed radiography machine. While cost-effective, this mechanism was associated with delays in care and was replaced with a cellular modem to send images to a central archive, which reliably delivers the images for reading by radiologists at Moi Teaching and Referral Hospital, using a web-based application, referralPACS. ${ }^{15}$ Radiologists report using a form that captures discrete data elements about each radiograph and referralPACS generates a PDF report that is printed centrally and distributed to each site within 2 weeks of the radiograph (figures 3 and 4). The results are also uploaded to an online results portal through a secure socket shell tunnel. Our web page uses hypertext transfer protocol secure (HTTPS) encryption for all interpretations. All results are filed in the patient's medical record.

Laboratory services, including testing for pregnancy (urine human chorionic gonadotropin), meningitis (cryptococcal antigen), TB (lateral flow urine lipoarabinomannan assay) and a serum basic metabolic profile are also available to patients, with or without an appointment, whenever the MDU is at the clinic. All laboratory services are performed by a certified laboratory technician employed by AMPATH and offered at the pointof-care, which enables results to be immediately made 


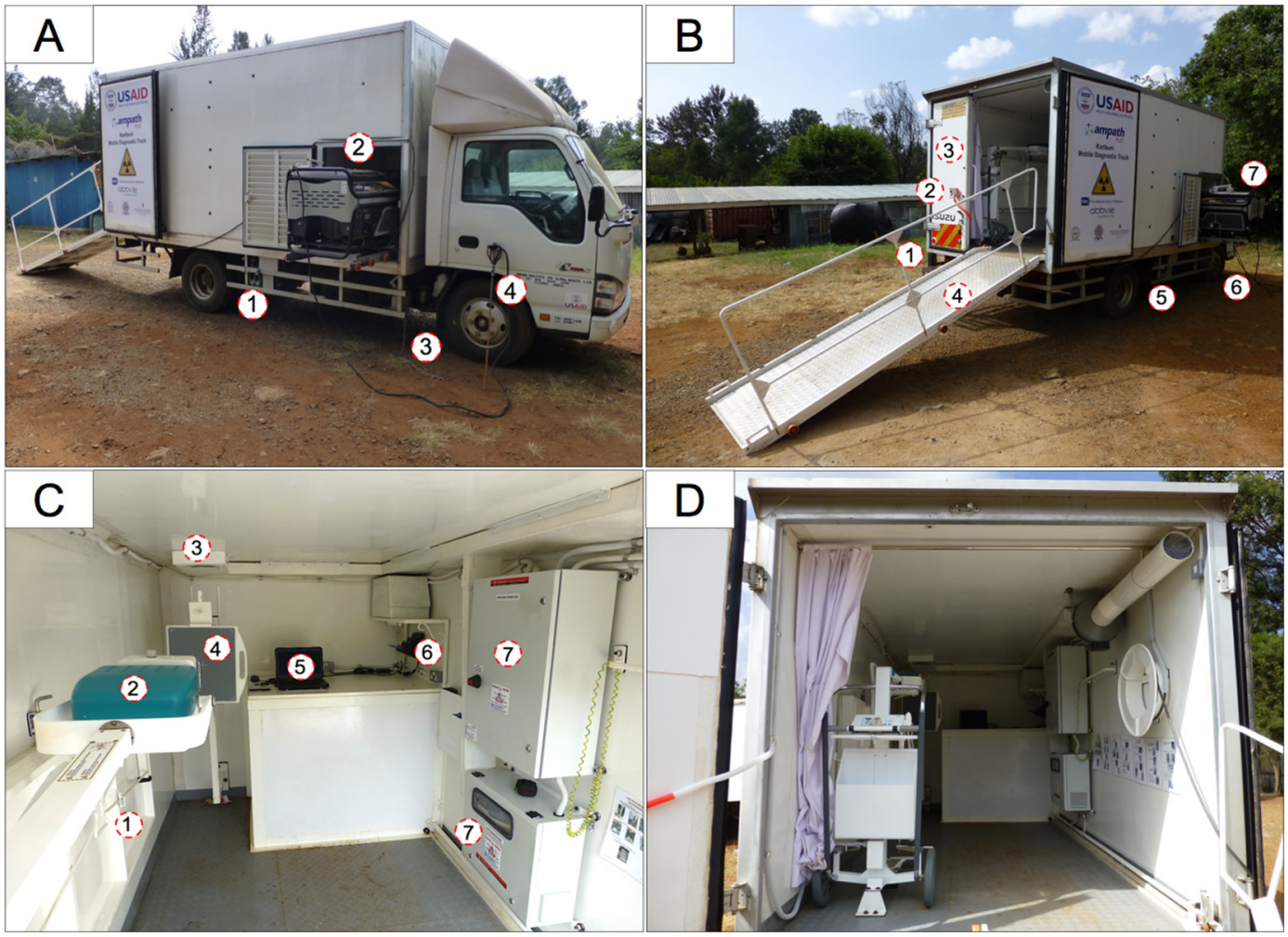

Figure 2 Mobile diagnostic unit equipment placement and descriptions. (A) Anterior side view: (1) fuel can; (2) slide-out electrical generator; (3) grounding chain; (4) grounding rod (hammered into the ground at each site). (B) Posterior side view: (1) standing platform for radiologists; (2) power factor correction device; (3) shielded door for radiologists to stand behind; (4) patient access ramp; (5) onboard generator fuel can; (6) redundant grounding devices (installed at each site); (7) slide-out electrical generator. (C) (interior view, detailed): (1) fold-down bed for supine radiographs; (2) X-ray generator; (3) negative pressure ventilation; (4) digital radiography cassette; (5) radiography laptop computer; (6) onboard $230 \mathrm{~V}$ power and charging; (7) power control, safety and distribution systems. (D) Interior view, general layout, shown with shielding door open.

available to the patient and clinician at the time they are performed.

\section{IMPLEMENTATION BENEFITS}

Our experience supports the feasibility of an MDU equipped with radiographic and basic laboratory services to address the major access gap for these health services

\begin{tabular}{|c|c|c|}
\hline Equipment & KSH & US\$ \\
\hline Truck chassis & 2850000 & 33139.53 \\
\hline Truck body & 1250000 & 14534.88 \\
\hline Outfitting truck body & 556000 & 6465.12 \\
\hline Engineering cost & 495000 & 5755.81 \\
\hline Radiation testing and certification & 60000 & 697.67 \\
\hline Electrical generator & 226000 & 2627.91 \\
\hline Sedecal Dragon (X-ray generator) & & 10950.00 \\
\hline Digital radiography & & 52100.00 \\
\hline Dell server & & 6182.00 \\
\hline & & 132452.92 \\
\hline
\end{tabular}

All costs in Kenyan shillings (KSH) were spent locally. Exchange rate: $86 \mathrm{KSH} / \mathrm{US} \$$. in rural areas of Kenya. Moreover, we identify three key successes with the MDU: a high detection rate of abnormal radiographic studies, improved access to radiology/

Table 2 Monthly maintenance costs for mobile diagnostic unit

\begin{tabular}{|c|c|c|}
\hline Expense & $\mathrm{KSH} /$ month & $\begin{array}{l}\text { US\$ (86 } \\
\text { KSH/US\$) }\end{array}$ \\
\hline Radiographer meal allowance & 6000 & \\
\hline Generator fuel & 10000 & \\
\hline Tires & 9000 & \\
\hline Inspection & 812 & \\
\hline Vehicle insurance & 6375 & \\
\hline Vehicle maintenance & 8400 & \\
\hline Fuel & 20000 & \\
\hline Tracking system & 1700 & \\
\hline Technology (CDs) & 8000 & \\
\hline Salaries & 100000 & \\
\hline \multirow[t]{2}{*}{ Equipment maintenance } & 10000 & \\
\hline & 180287 & 2096.36 \\
\hline
\end{tabular}

$\mathrm{KSH}$, Kenyan shilling. 


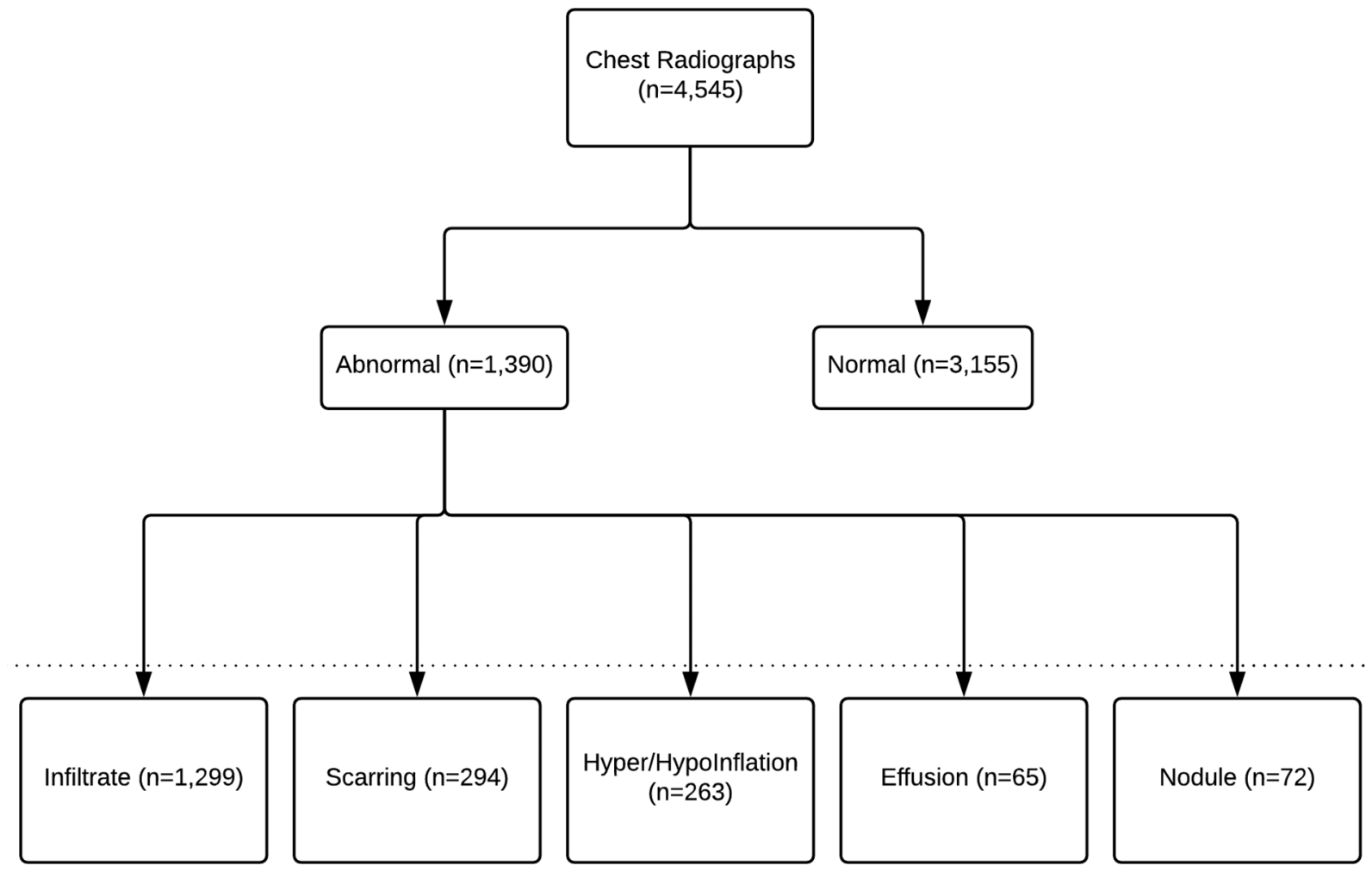

Figure 5 Description of X-ray results. Abnormality classes are not exclusive, patients may have one or more.

laboratory services and increased patient engagement in these services.

\section{Laboratory findings}

As of June 2017, 523 laboratory services had been provided. Pregnancy tests accounted for 183 of the MDU laboratory services, followed by basic metabolic profile (169), cryptococcal antigen (98) and rapid urine lipoarabinomannan antigen (73).

\section{High detection rates}

From April 2014 to April 2018, 4545 chest radiographs were performed by the MDU, ranging from 10 to 20 to 40-50 radiographs per day at low-volume and highvolume facilities, respectively. All radiographs were interpreted by radiologists with board-certified Kenyan medical licenses. Most X-rays performed were usable according to the radiographer's assessment at the time of the procedure; those that were deemed unusable were simply repeated given the digital format of the film. Of these, $3155(69 \%)$ were normal and $1390(31 \%)$ were abnormal, demonstrating a spectrum of pathological findings (figure 5). The number of patients receiving chest radiographs each month has risen steadily each month since implementation. Our rate of detection was much higher than anticipated, as more than $30 \%$ of chest radiographs taken were abnormal. It is likely that many of these abnormalities would not have been detected in the absence of the MDU, as the areas the MDU serves do not otherwise have access to diagnostic testing. Our results also demonstrate the potential impact of the MDU in improving detection of $\mathrm{TB}$ and other pathologies in resource-constrained settings.

\section{Improved access}

The MDU was developed as part of AMPATH's population health strategy and seeks to increase access to radiography and laboratory services, but not to take the place of permanent healthcare facilities. Although we identified these services as priorities for the patient population at AMPATH, it is important to note that the MDU platform can accommodate multiple potential diagnostic and treatment modalities to increase access to healthcare services in resource-limited settings. Ultrasound and CT scan, for example, have been incorporated into MDUs in other settings. ${ }^{16}{ }^{17}$ While AMPATH is still focused on increasing infrastructure that allows its healthcare facilities to function independently of the MDU, one of the major successes of the MDU is the ability to provide basic radiographs to a population living in remote areas. Many of the facilities the MDU services are several kilometres away from major highways and are accessible only by dirt road. Having an independent steady power source from our generator improves reliability, and digital radiography reduces cost through elimination of film and processing supplies. Furthermore, acquisition of film and processing supplies is not trivial in Kenya, and frequently leads to extended downtimes at other facilities. In addition, patients who use the MDU or seek out AMPATH services because of the services the MDU provides gain access to AMPATH's larger network of healthcare services 
Verified: true

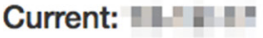

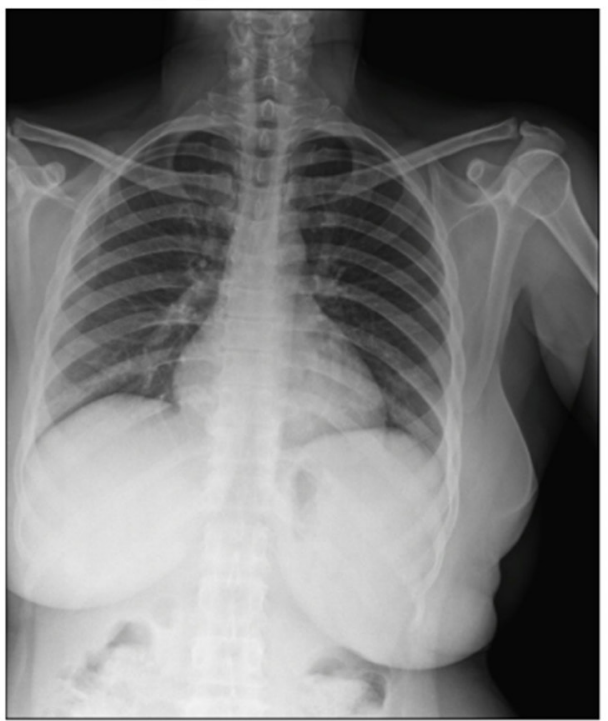

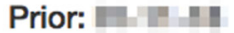

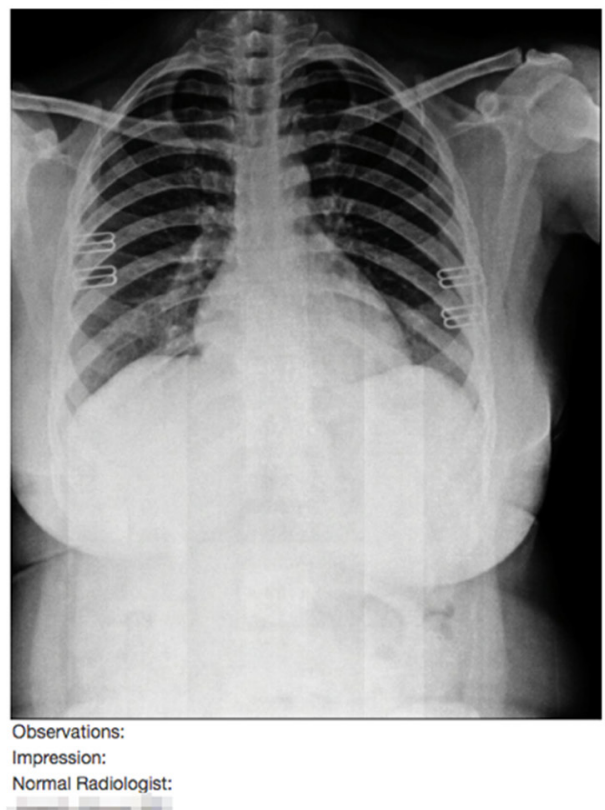

Priors

Pnt

\section{Clinical Summary}

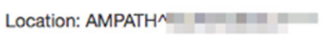

Indication: chest pain

Normal Full Report Reject Encounter

Figure 3 Example of web-page layout for radiologist interpretation. Prior films acquired on the mobile diagnostic unit are available for comparison. A shortcut button to create a normal report is important for workflow due to the large number of normal radiographs. See figure 4 for the full report form.

and programme as AMPATH patients, contrasting with companies aiming to replicate healthcare facilities through broadly capable mobile units. ${ }^{18}$ In so doing, the AMPATH MDU takes a less costly and more efficient approach that synergises with its existing infrastructure.

\section{Patient engagement}

Increased patient engagement was a benefit we were not expecting. Several of our clinicians anecdotally reported that patients are excited about access to new technology, and curious about engaging with radiographic and laboratory services, resulting in an increase in the number of patients visiting the health facilities. Similar findings were reported using MDUs for TB active case detection as well elsewhere in sub-Saharan Africa. ${ }^{7}$ This finding is important because patients who live long distances from tertiary hospitals access lower-level care locally and avoid travelling until they have advanced disease. Concerning $\mathrm{TB}$, moving diagnostics closer to the patient has the potential to decrease TB burden by increasing screening chest radiographs especially for patients with newly diagnosed HIV and those who have not previously had a chest X-ray. ${ }^{1314}$ Such screening should lead to earlier diagnosis and treatment. This is particularly important in Kenya, where an estimated $40 \%$ of TB cases are currently undetected. ${ }^{13}$

\section{CHALLENGES AND LIMITATIONS Implementation}

Although the design of the MDU came with challenges (see Design section), the majority of challenges we encountered were during the implementation of the MDU. A primary radiologist reading, which is the standard of care throughout the USA, is challenging in Kenya due to multiple factors including poor internet connectivity, too few radiologists and delivery time for paper reports. Poor internet connectivity in rural areas creates delays in archiving images for radiologist review and precludes clinicians' access to the online results portal. To address these issues, we have ensured that the local clinic has a copy of each radiograph for immediate review by clinical officers. To increase immediate access to radiologist readings, efforts are ongoing to allow synchronisation of the imaging archive system with AMPATH's electronic medical record system to facilitate clinicians' access to images and formal reports at the point of care. ${ }^{19}$ We are also actively developing a computer-aided detection (CAD) system, in partnership with the National Institutes of Health, which aims to identify which radiographs contain abnormalities that need to be evaluated quickly. This CAD system is currently being implemented on the MDU (figure 6). Other possible solutions to increase 


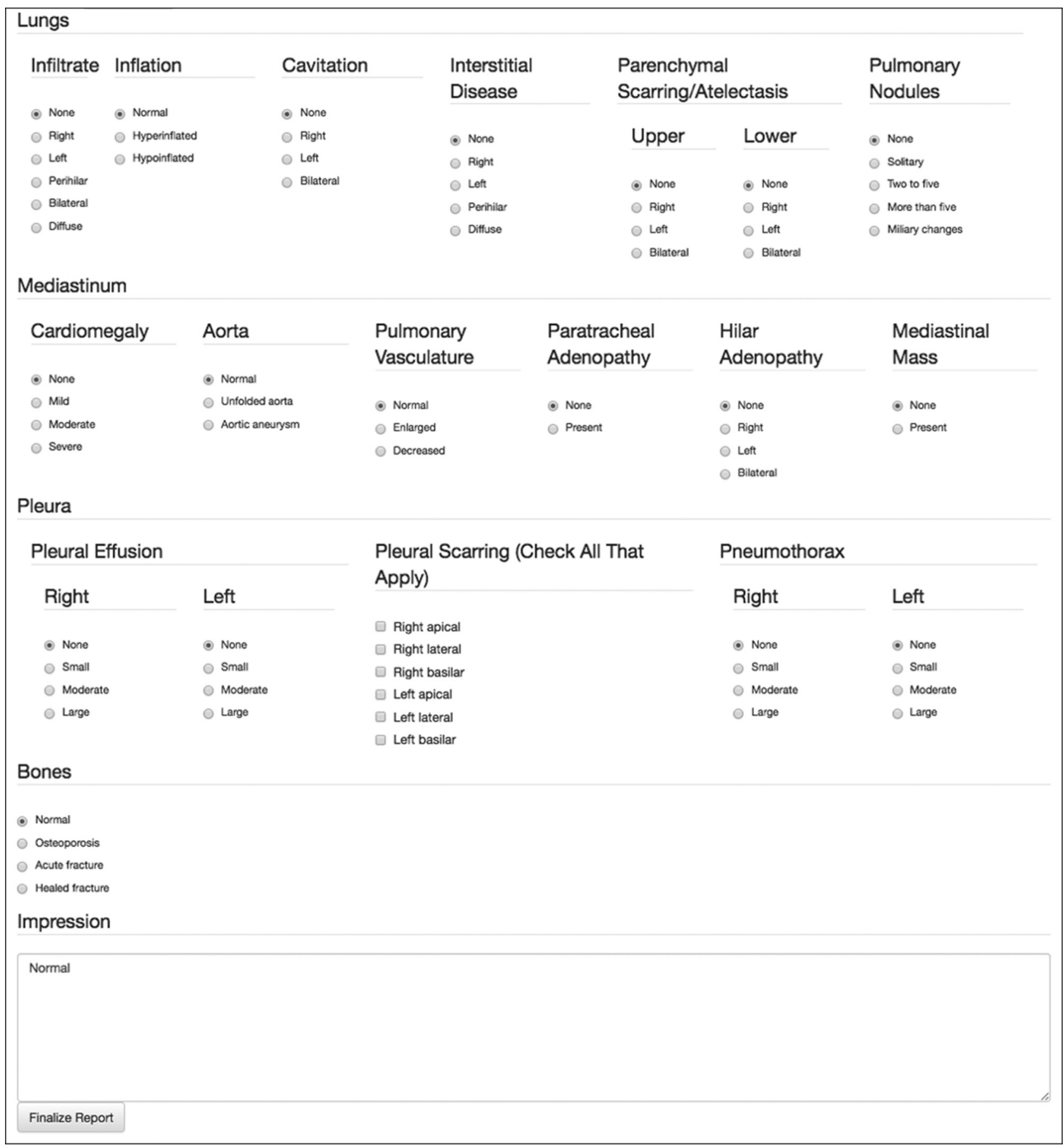

Figure 4 Report form for abnormal radiographs. Data elements are captured discreetly in addition to a free-text impression.

efficiency include task shifting to clinical officers with escalation of more complex images to be read by a radiologist. Outsourcing or telemedicine are additional potential solutions but are also limited by Internet connectivity and significantly higher costs.

\section{Sustainability}

Funding for equipment maintenance to continue providing radiographs is a challenge. Currently, all radiographs and laboratory tests performed by the MDU, except the basic metabolic profile, are offered free of charge due to various AMPATH funding partners. To ensure long-term sustainability of the MDU programme and other healthcare initiatives, AMPATH is focusing on enrolling patients in the National Hospital Insurance Fund, a new government insurance programme to cover healthcare costs for Kenyans. Vouchers and conditional cash transfers are other methods used to increase access to healthcare services in sub-Saharan Africa. ${ }^{20}$ Although the early diagnosis of TB and other communicable diseases is likely to have benefits in terms of patient morbidity and mortality and prevention of ongoing disease transmission in the community, we are unable 


\section{STATUS}

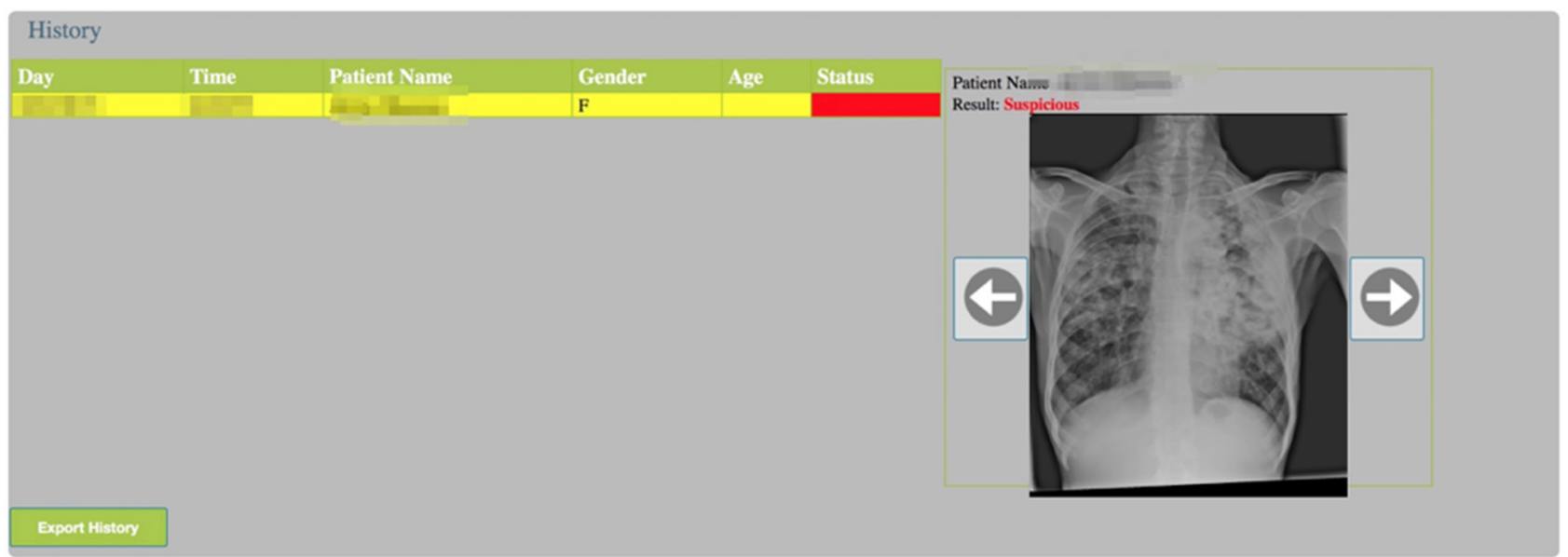

Figure 6 Example of the National Library of Medicine (NLM) computer-aided detection system. The system confers either red or green status to each chest radiograph, shortly after acquisition.

to characterise the cost-effectiveness of the MDU given the lack of these patient-level clinical and outcomes data. However, the MDU programme requires that at least 10 patients to be scheduled for an X-ray at a given site for the unit to attend that site; once on site, the cost per X-ray decreases with each additional X-ray performed. Finally, we do not have data on the clinical treatment courses for patients who received X-rays and laboratory tests through our MDU and the extent to which the MDU interventions influenced patient outcomes and clinical decision-making. This information is also important in understanding the true impact and cost-effectiveness of the MDU intervention.

\section{FUTURE DIRECTIONS}

Future efforts are planned to further characterise the impact of the MDU on tuberculosis diagnosis and control in the catchment area, as well as the cost-effectiveness of the MDU strategy in comparison with other strategies to scale laboratory and radiological resources in our setting.

\section{CONCLUSIONS}

The MDU provides a potential solution to resource-limited, rural areas seeking to increase access to laboratory and radiology resources in western Kenya. The MDU may serve as a model for other settings facing similar challenges, both to address the burden of rural TB and the growing need for non-communicable disease detection and management in the population.

Contributors The research and development for aspects of this project was supported by the Intramural Research Program of the National Library of Medicine, National Institutes of Health. The design, equipment and operation of the MDU is supported by a grant from the AbbVie Foundation. This research has also been supported by the President's Emergency Plan for AIDS Relief (PEPFAR) through USAID under the terms of Cooperative Agreement No. AID-623-A-12-0001. It is made possible through joint support of the United States Agency for International Development (USAID).

Funding The authors have not declared a specific grant for this research from any funding agency in the public, commercial or not-for-profit sectors.

Disclaimer The contents are the sole responsibility of AMPATH and do not necessarily reflect the views of USAID or the United States Government.

Competing interests None declared.

Patient consent Not required.

Ethics approval Moi University/Moi Teaching and Referral Hospital Institutional Research and Ethics Committee.

Provenance and peer review Not commissioned; externally peer reviewed.

Data statement No additional data are available.

Open access This is an open access article distributed in accordance with the Creative Commons Attribution Non Commercial (CC BY-NC 4.0) license, which permits others to distribute, remix, adapt, build upon this work non-commercially, and license their derivative works on different terms, provided the original work is properly cited, appropriate credit is given, any changes made indicated, and the use is non-commercial. See: http://creativecommons.org/licenses/by-nc/4.0/

\section{REFERENCES}

1. World Bank Group, 2017. Rural population (\% of total population). https://data.worldbank.org/indicator/SP.RUR.TOTL.ZS (accessed 15 Feb 2018).

2. Kawooya MG. Training for rural radiology and imaging in subsaharan Africa: addressing the mismatch between services and population. J Clin Imaging Sci 2012;2:37.

3. Kawooya MG, Pariyo G, Malwadde EK, et al. Assessing the diagnostic imaging needs for five selected hospitals in Uganda. $J$ Clin Imaging Sci 2011;1:53.

4. World Health Organization. Chest radiography in tuberculosis detection: summary of current who recommendations and guidance on programmatic approaches. Geneva: WHO, 2016.

5. World Health Organization. Global tuberculosis report. Geneva: WHO, 2016. 
6. Golub JE, Mohan $\mathrm{Cl}$, Comstock GW. Active case finding of tuberculosis: historical perspective and future prospects. Int $J$ Tuberc Lung Dis 2005;9:1183-203.

7. Corbett EL, Bandason T, Duong T, et al. Comparison of two active case-finding strategies for community-based diagnosis of symptomatic smear-positive tuberculosis and control of infectious tuberculosis in Harare, Zimbabwe (DETECTB): a cluster-randomised trial. Lancet 2010;376:1244-53.

8. Heuvelings CC, de Vries SG, Greve PF, et al. Effectiveness of interventions for diagnosis and treatment of tuberculosis in hardto-reach populations in countries of low and medium tuberculosis incidence: a systematic review. Lancet Infect Dis 2017;17:e144-58.

9. Inui TS, Nyandiko WM, Kimaiyo SN, et al. AMPATH: living proof that no one has to die from HIV. J Gen Intern Med 2007;22:1745-50.

10. AMPATH, 2018. AMPATH: leading with care. http://www. ampathkenya.org/ (accessed 20 Feb 2018).

11. Kenya National Bureau of Statistics. Kenya-2009 Kenya Population and Housing Census. Kenya, 2009.

12. National AIDS Control Council. Busia county HIV \& AIDS strategic plan. Busia County, Kenya, 2014.

13. Republic of Kenya: Ministry of Health. Kenya tuberculosis prevalence survey. Nairobi: Kenya, 2016.
14. World Health Organization. Guidelines for intensified tuberculosis case-finding and isoniazid preventive therapy for people living with HIV in resource-constrained settings. Geneva: WHO, 2011.

15. Shadowdoc, 2018. Shadowdoc/referralpacs. https://github.com/ shadowdoc/referralpacs (accessed 29 Jun 2018).

16. Sone S, Takashima S, Li F, et al. Mass screening for lung cancer with mobile spiral computed tomography scanner. Lancet 1998;351:1242-5.

17. Chavez MA, Naithani N, Gilman RH, et al. Agreement between the World Health Organization algorithm and lung consolidation identified using point-of-care ultrasound for the diagnosis of childhood pneumonia by general practitioners. Lung 2015;193:531-8.

18. OneStopTB clinic, 2018. Delft imaging systems. https://www.delft. care/onestoptb/ (accessed 29 Jun 2018)

19. Tierney WM, Rotich JK, Hannan TJ, et al. The AMPATH medical record system: creating, implementing, and sustaining an electronic medical record system to support HIV/AIDS care in western Kenya. Stud Health Technol Inform 2007;129(Pt 1):372-6.

20. Owusu-Addo E, Renzaho AMN, Smith BJ. Evaluation of cash transfer programs in sub-saharan Africa: a methodological review. Eval Program Plann 2018;68:47-56. 